\title{
Comparação entre cirurgia e aplicações unilaterais e bilate- rais de toxina botulínica para 0 tratamento dos estrabismos
}

\author{
Comparison between surgery and unilateral and bilateral injections of botulinum toxin to \\ treat strabismus
}

Maria de Lourdes M. M. Villas Boas ${ }^{1}$

Henderson Celestino de Almeida ${ }^{2}$

Hospital São Geraldo da Universidade Federal de Minas Gerais e Instituto de Olhos de Belo Horizonte.

${ }^{1}$ Doutora em Oftalmologia e Professora voluntária do Departamento de Estrabismo do Hospital São Geraldo da Universidade Federal de Minas Gerais. Chefe do Serviço de Estrabismo do Instituto Mineiro de Olhos e do Instituto da Visão.

${ }^{2}$ Professor titular no Departamento de Oftalmo/ Otorrinolaringologia da Faculdade de Medicina da Universidade Federal de Minas Gerais. Chefe do Serviço de Estrabismo do Hospital São Geraldo.

Endereço para correspondência: Dra. Maria de Lourdes Motta Moreira Villas Boas - Rua Ceará, 1333/201 Funcionários Belo Horizonte (MG) CEP 30150-311. E-mail:mlvboas@uol.com.br

INTRODUÇÃO

Quando o tratamento clínico para o estrabismo (óculos, prismas, mióticos, exercícios ortópticos) não está indicado ou falha em promover um adequado alinhamento ocular, há indicação para cirurgia. Embora quase sempre bem sucedida, ela envolve hospitalização, anestesia, cortes, suturas e afastamento temporário da escola ou do trabalho.

Na década de 80, Scott introduziu uma forma alternativa de tratamento através da utilização da toxina botulínica ${ }^{(1)}$. Ele começou a utilizá-la em seres humanos em 1977. Em 1982, ela passou a ser utilizada, ainda de forma experimental, por vários pesquisadores em todo o mundo, sob a sua orientação. E, finalmente, em 1989, a toxina foi liberada pelo FDA (Food and Drug Administration) para o tratamento do blefaroespasmo e do estrabismo.

Observando os trabalhos publicados sobre este assunto, constatamos que, no nosso meio, apenas dois autores relataram suas experiências de tratamento do estrabismo com a toxina botulínica: Souza Dias ${ }^{(2)}$ e Almeida Santos $^{(3-4)}$. Além disso, não encontramos qualquer trabalho que comparasse os efeitos do tratamento unilateral e bilateral. Essa é uma polêmica antiga e muito bem estudada em inúmeros trabalhos no caso do tratamento 
cirúrgico dos estrabismos. Mesmo a comparação entre a eficácia do tratamento com a toxina botulínica e cirurgia, feita de maneira aleatória, só foi descrita em um trabalho ${ }^{(5)}$.

Por isso, o objetivo do presente trabalho foi comparar a eficácia das três formas de tratamento (aplicações unilaterais e bilaterais de toxina botulínica e a cirurgia) quanto ao percentual de correção do desvio ocular e ao índice de sucesso terapêutico (definido como desvio residual de até 10 DP).

\section{MÉTODOS}

Neste trabalho foram estudados 97 pacientes provenientes de hospital universitário e da clínica particular da autora.

O critério de inclusão foi o tamanho do desvio ocular, que variou de 15 a $30 \mathrm{DP}$.

Os pacientes foram divididos em três grupos, de acordo com o tratamento efetuado. Os dois primeiros grupos receberam aplicação da toxina botulínica, tendo sido feita distribuição aleatória para injeção unilateral e bilateral.

A divisão dos grupos ficou constituída como mostra o quadro abaixo.

Para o objetivo do presente trabalho, que era o de observar o efeito da aplicação da toxina no tamanho do desvio ocular, os grupos foram considerados comparáveis, pois as diferenças na idade média dos pacientes e no tipo de estrabismo não interferiram nesse efeito. $\mathrm{O}$ tamanho dos desvios pré-tratamento e o número de pacientes em cada grupo também foram considerados comparáveis pela análise estatística.

A toxina botulínica utilizada foi a forma liofilizada, congelada, do tipo A, na concentração de 0,05 microgramas em 500 microgramas de seroalbumina. Cada frasco contém $100 \mathrm{U}$ de toxina. Ela era conservada congelada a -5 graus centígrados. Imediatamente antes do uso, era diluída com soro fisiológico em quantidade que variava com a concentração desejada para tratamento. Essa concentração dependia do tamanho do desvio. Em 1 paciente, com esotropia de $15 \mathrm{DP}$ e ambliopia profunda em um dos olhos, foi utilizada a concentração de $1,25 \mathrm{U}$

\begin{tabular}{|c|c|c|c|}
\hline \multicolumn{4}{|c|}{ QUADRO } \\
\hline \multirow[b]{2}{*}{$\begin{array}{l}\text { Forma de } \\
\text { tratamento }\end{array}$} & \multicolumn{3}{|c|}{ GRUPOS } \\
\hline & $\begin{array}{c}\text { I } \\
\text { Aplicação } \\
\text { unilateral }\end{array}$ & $\begin{array}{c}\text { II } \\
\text { Aplicação } \\
\text { bilateral }\end{array}$ & $\begin{array}{c}\text { III } \\
\text { Cirurgia }\end{array}$ \\
\hline $\begin{array}{l}\text { Número de } \\
\text { pacientes }\end{array}$ & 44 & 24 & 29 \\
\hline $\begin{array}{l}\text { Distribuição } \\
\text { por sexo }\end{array}$ & $\begin{array}{l}21 \text { mulheres } \\
23 \text { homens }\end{array}$ & $\begin{array}{l}11 \text { mulheres } \\
13 \text { homens }\end{array}$ & $\begin{array}{l}13 \text { mulheres } \\
16 \text { homens }\end{array}$ \\
\hline $\begin{array}{l}\text { Distribuição } \\
\text { por idade }\end{array}$ & $\begin{array}{c}5 \text { a } 73 \text { anos } \\
\text { média }=31,1 \\
\text { mediana }=23,5\end{array}$ & $\begin{array}{c}10 \text { a } 73 \text { anos } \\
\text { média }=25,3 \\
\text { mediana }=22,5\end{array}$ & $\begin{array}{c}4 \text { a } 37 \text { anos } \\
\text { média }=15,6 \\
\text { mediana }=14,0\end{array}$ \\
\hline $\begin{array}{l}\text { Tipo de } \\
\text { estrabismo }\end{array}$ & $\begin{array}{l}\text { convergente } \\
=33 \\
\text { divergente } \\
=11\end{array}$ & $\begin{array}{l}\text { convergente } \\
=23 \\
\text { divergente } \\
=01\end{array}$ & $\begin{array}{c}\text { convergente } \\
=29 \\
\text { divergente } \\
=0\end{array}$ \\
\hline
\end{tabular}

(toxina diluída com $8 \mathrm{ml}$ de soro fisiológico); em 7 pacientes, com desvios que variaram entre 15 e $20 \mathrm{DP}$, foi utilizada a concentração de 2,5 U (toxina diluída com $4 \mathrm{ml}$ de soro fisiológico); em 1 paciente, com esotropia de $30 \mathrm{DP}$ e paralisia do reto lateral foi utilizada a concentração de 10,0 U (toxina diluída com $1 \mathrm{ml}$ de soro) no reto medial; e nos demais pacientes foi utilizada a concentração de 5,0 U (diluição feita com $2 \mathrm{ml}$ de soro), todos com desvio entre 20 e 30 DP.

Em 2 pacientes foi feita sedação com Ketamina $(1,5 \mathrm{ml} / \mathrm{kg}$ de peso corporal) por se tratar de crianças com menos de 10 anos de idade. Em todos os demais pacientes a aplicação foi ambulatorial, sob anestesia tópica.

Em todos os pacientes, a rotina foi a seguinte:

- Medida do desvio ocular, para longe e perto, utilizando-se o método de cobertura alternada com prismas, nos pacientes com fixação central, e o método de Krimsky naqueles com fixação excêntrica.

- Anestesia tópica, com 5 gotas de colírio anestésico (Cloridrato de Proximetacaína 0,5\%), exceto naqueles que se submeteram à sedação com Ketamina, no bloco cirúrgico, sob supervisão de um anestesista.

- Administração de 1 gota de colírio de Fenilefrina a 10\%, para promover uma vasoconstrição.

- Diluição da toxina, através da injeção da quantidade desejada de soro fisiológico.

- Aspiração de $0,1 \mathrm{ml}$ da solução da toxina botulínica tipo A na seringa de $1 \mathrm{ml}$, que era acoplada ao eletromiógrafo. (Foi utilizado o tipo portátil, desenvolvido por Scott, especialmente para a aplicação de toxina).

- Colocação de um eletrodo de eletrocardiograma, tipo infantil, na região frontal do paciente e posterior acoplamento desse eletrodo também ao eletromiógrafo.

- Colocação de um blefarostato.

- Utilização de um compasso de estrabismo para medir a posição presumida da inserção escleral do músculo a ser injetado, principalmente naqueles pacientes que já tinham sofrido recuos musculares.

- Introdução da agulha a partir desse local medido, após ter sido solicitado ao paciente que olhasse em direção oposta ao campo de ação do músculo alvo. No caso das crianças, nas quais a injeção foi feita sob sedação, a movimentação do olho era feita com pinça.

- Verificação da posição da agulha, solicitando-se ao paciente que olhasse em direção ao campo de ação do músculo alvo, prestando-se atenção ao sinal sonoro emitido pelo eletromiógrafo quando a agulha se encontrava posicionada dentro do corpo muscular, a pelo menos 2,5 mm da sua inserção.

- Era feita, a seguir, a injeção lenta da toxina, com posterior retirada da agulha e liberação do paciente.

- Retornos para controle eram feitos nos intervalos de 7,30 e 90 dias, quando eram repetidas as medidas do desvio ocular. 


\section{RESULTADOS}

Para comparar as medidas dos desvios, utilizou-se o teste de Kruskal-Wallis. A comparação entre a evolução das medidas em cada grupo, ao longo do tempo, foi feita utilizando-se o teste de Friedman, e para avaliar os índices de sucesso terapêutico foi utilizado o teste do qui-quadrado.

Todos os resultados foram considerados significativos a um nível de significância de $5 \%(\mathrm{p}<0,05)$. Os resultados quanto ao percentual de correção do desvio ocular horizontal, para longe e perto, podem ser vistos nas tabelas de 1 a 4 , e os resultados quanto aos índices de sucesso terapêutico, nos gráficos $1 \mathrm{e} 2$. As figuras de 1 a 4 exemplificam casos comitantes e paralíticos, tratados com injeções uni e bilaterais.

\section{DISCUSSÃO}

A idéia de injetar um agente farmacológico no músculo extraocular, com o objetivo de produzir uma paralisia temporária, foi de Berens, conforme citado por Scott et al. ${ }^{(6)}$. A substância por ele utilizada foi o álcool, que não se mostrou eficaz.

Foi Jampolsky quem também, segundo $\operatorname{Scott}^{(6)}$, em 1963,

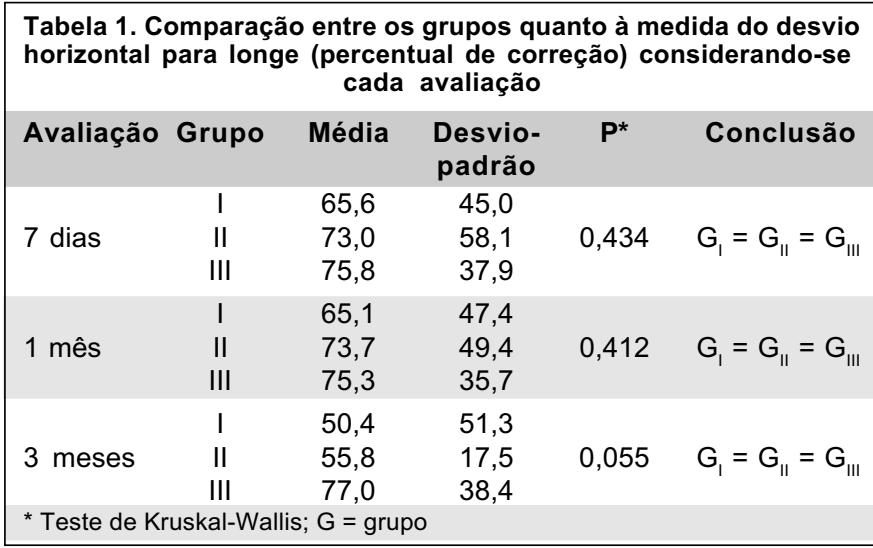

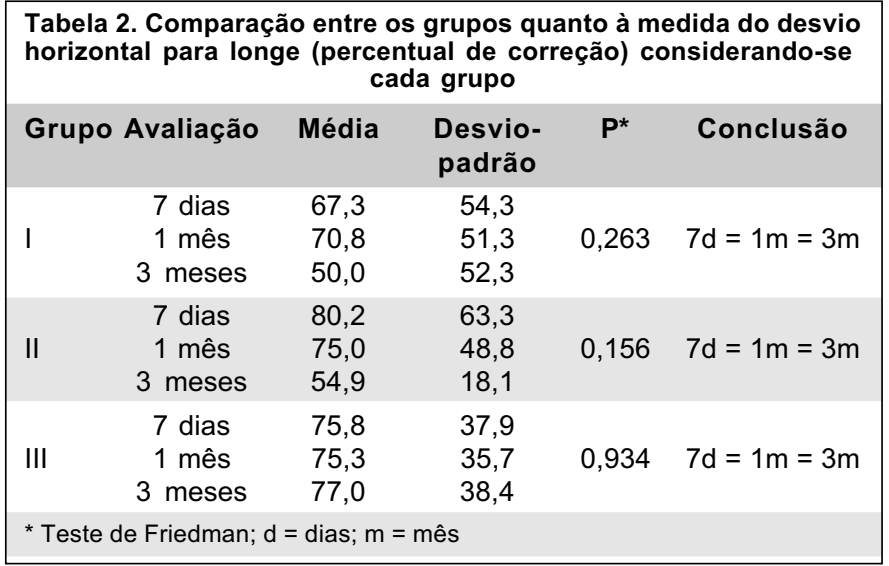

\begin{tabular}{|c|c|c|c|c|c|}
\hline Avaliação & Grupo & Média & $\begin{array}{l}\text { Desvio- } \\
\text { padrão }\end{array}$ & $P^{*}$ & Conclusão \\
\hline 7 dias & $\begin{array}{c}\text { I } \\
\text { II } \\
\text { III }\end{array}$ & $\begin{array}{l}60,8 \\
67,4 \\
70,1\end{array}$ & $\begin{array}{l}62,1 \\
48,6 \\
36,1\end{array}$ & 0,535 & $G_{1}=G_{\| 1}=G_{I I I}$ \\
\hline 1 mês & $\begin{array}{c}\text { I } \\
\text { II } \\
\text { III }\end{array}$ & $\begin{array}{l}61,7 \\
70,2 \\
68,4\end{array}$ & $\begin{array}{l}63,4 \\
41,4 \\
26,8\end{array}$ & 0,535 & $\mathrm{G}_{1}=\mathrm{G}_{\|}=\mathrm{G}_{\| I I}$ \\
\hline 3 meses & $\begin{array}{l}\text { I } \\
\text { II } \\
\text { III }\end{array}$ & $\begin{array}{l}48,6 \\
49,2 \\
72,8\end{array}$ & $\begin{array}{l}66,5 \\
19,6 \\
38,7\end{array}$ & 0,091 & $G_{1}=G_{\| I}=G_{I I I}$ \\
\hline
\end{tabular}

\begin{tabular}{|c|c|c|c|c|c|}
\hline Grupo & Avaliação & Média & $\begin{array}{l}\text { Desvio- } \\
\text { padrão }\end{array}$ & $\mathbf{P}^{*}$ & Conclusão \\
\hline I & $\begin{array}{c}7 \text { dias } \\
1 \text { mês } \\
3 \text { meses }\end{array}$ & $\begin{array}{l}60,7 \\
69,5 \\
48,9\end{array}$ & $\begin{array}{l}75,7 \\
70,5 \\
67,8\end{array}$ & 0,103 & $7 d=1 m=3 m$ \\
\hline II & $\begin{array}{c}7 \text { dias } \\
1 \text { mês } \\
3 \text { meses }\end{array}$ & $\begin{array}{l}72,3 \\
73,6 \\
50,0\end{array}$ & $\begin{array}{l}52,7 \\
38,9 \\
21,4\end{array}$ & 0,065 & $7 d=1 m=3 m$ \\
\hline III & $\begin{array}{c}7 \text { dias } \\
1 \text { mês } \\
3 \text { meses }\end{array}$ & $\begin{array}{l}69,0 \\
68,0 \\
72,8\end{array}$ & $\begin{array}{l}36,3 \\
27,2 \\
38,7\end{array}$ & 0,825 & $7 d=1 m=3 m$ \\
\hline
\end{tabular}

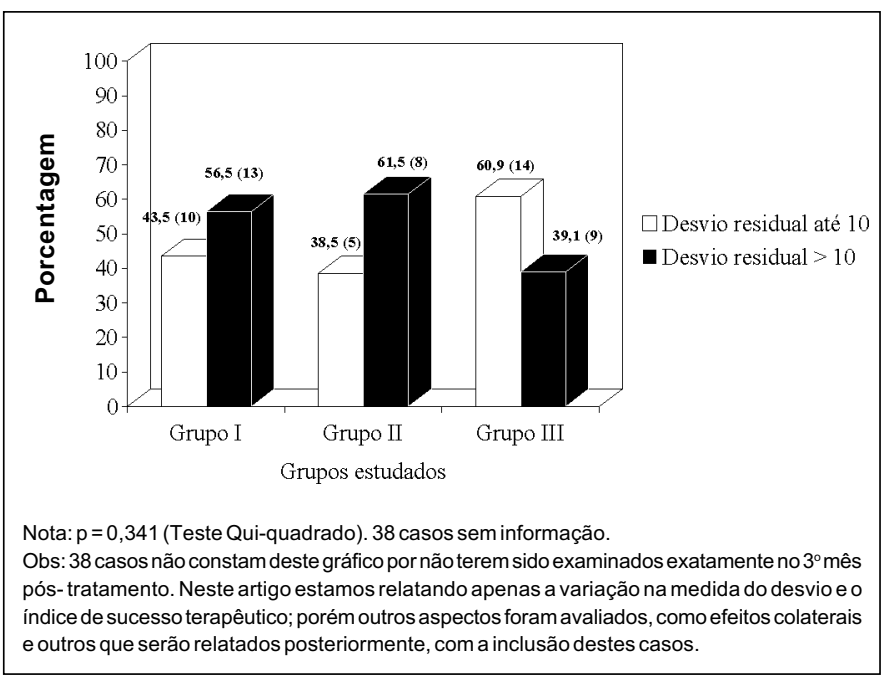

Gráfico 1 - Avaliação dos grupos quanto ao desvio residual para longe no 3 o mês do tratamento

sugeriu um projeto para avaliar os efeitos de drogas injetadas com o auxílio de um eletromiógrafo, mas as experiências não se concretizaram por falta de um modelo animal. Os agentes anestésicos, como os barbitúricos e o halotano, aboliam o sinal eletromiográfico. Posteriormente o próprio $\mathrm{Scott}^{(6)} \mathrm{ob}-$ 


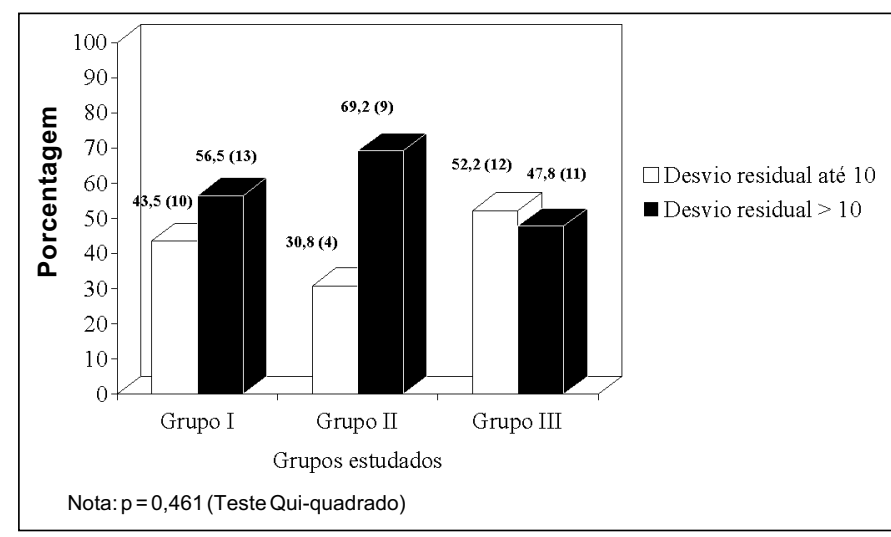

Gráfico 2 - Avaliação dos grupos quanto ao desvio residual para perto no $3^{\circ}$ mês do tratamento

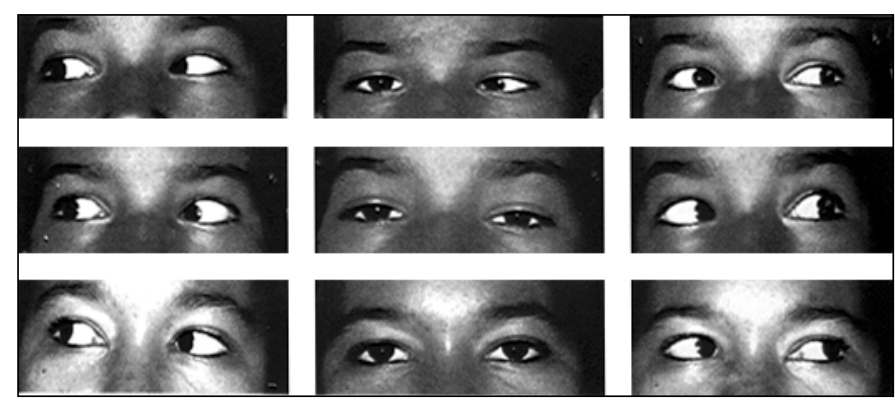

Figura 1 - Esotropia infantil. Primeira linha: pré-tratamento - ET 25 DP - ET' 25 DP; Segunda linha: 7 dias após aplicação de 5 U de TBA no músculo reto medial; Terceira linha: 2 anos após a aplicação E 2 DP - E' 3 DP
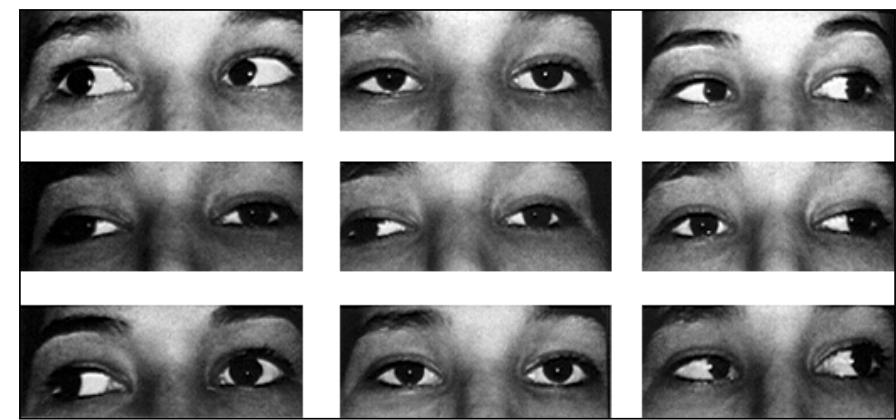

Figura 2 - Esotropia infantil. Primeira linha: pré-tratamento - ET 20 DP - ET' 20 DP; Segunda linha: 7 dias após aplicação de 5 U de TBA em ambos os músculos retos mediais - XT 30 DP - XT' 25 DP. Limitação da adução de -4 no OE e -3 no OD; Terceira linha: 3 meses após a aplicação - XT 10 DP - orto'

servou que a ketamina era capaz de anestesiar macacos e seres humanos, preservando o sinal eletromiográfico. Esse sinal, captado por uma agulha-eletrodo especial, ajudava a localizar o músculo para a aplicação da droga ${ }^{(7)}$. Depois de testar várias substâncias, chegou à conclusão de que a melhor tinha sido a toxina botulínica. Ela é uma potente neurotoxina produzida pela bactéria anaeróbica GRAM positiva Clostridium botulinum, que possui oito tipos imunologicamente distintos, que
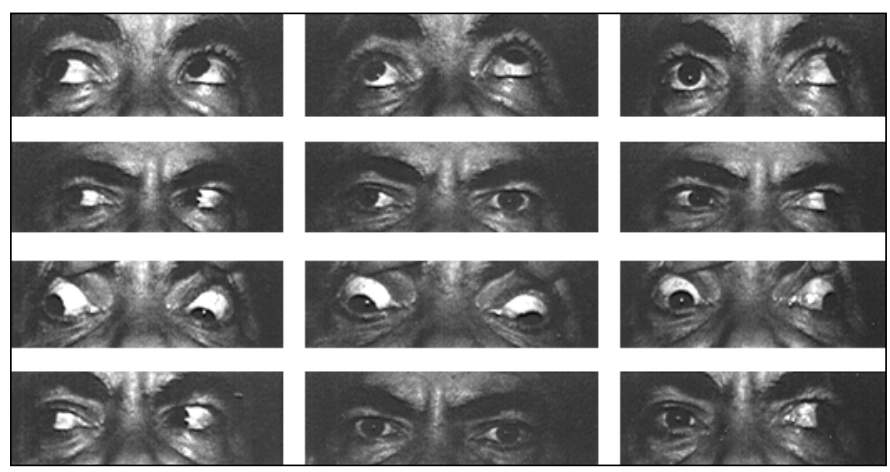

Figura 3 - Paresia traumática do III nervo craniano. Três primeiras linhas: pré-tratamento XT 30 + HT D 6 DP - XT' 30 + HT' D 6 DP; Última linha: 3 meses após aplicação de 2,5 U de TBA no músculo reto lateral direito - $E(T) 2+H(T)$ D 8 DP - E(T)' 4 + H(T)' D 12 DP
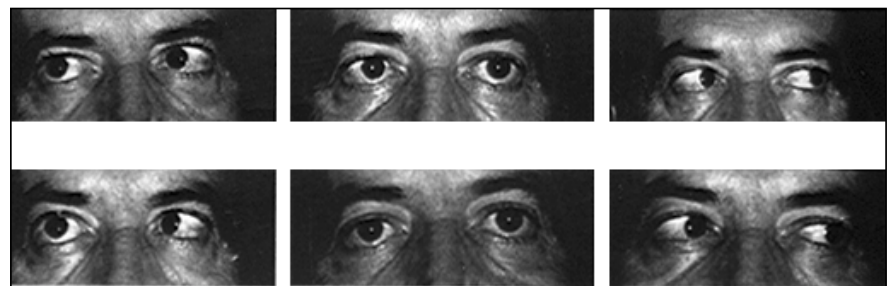

Figura 4 - Paralisia traumática do VI nervo craniano direito. Primeira linha: pré-tratamento - ET 25 DP - ET' 25 DP; Segunda linha: 7 dias após aplicação de $5 \mathrm{U}$ de TBA em ambos os músculos retos mediais - $E(T)$ 4 DP - orto'

são: $A, B, C_{1}, C_{2}, D, E, F$ e $G^{(8)}$. O tipo A é o mais utilizado nas preparações comerciais, por ser mais facilmente cristalizável de forma estável ${ }^{(1)}$.

O mecanismo de ação da toxina é um bloqueio nervoso présináptico por inibição da liberação de acetilcolina na placa motora. A toxina ocupa os sítios que seriam ocupados pelo íon cálcio na fibra colinérgica, evitando a exocitose da acetilcolina, que é cálciodependente. O músculo paralisado mostra histopatologicamente sinais de atrofia, e a porção nervosa terminal discretos sinais de alterações desmielinizantes, que subseqüentemente sofrem regeneração. ${ }^{(1)} \mathrm{O}$ tempo de duração do efeito é, em média, três meses, sendo que após esse tempo a eletromiografia do músculo injetado apresenta amplitude normal ${ }^{(6)}$.

O objetivo da aplicação da toxina no tratamento do estrabismo é promover uma paralisia adequada, porém temporária, do músculo injetado, para que ele se torne esticado e ligeiramente atrofiado, enquanto seu antagonista se torna encurtado e contraturado. Por esse processo, a posição do olho fica mais balanceada. Quando a aplicação está bem indicada, o alinhamento ocular permanece por anos após ter desaparecido a paralisia muscular. Por isso, a aplicação da toxina é considerada por alguns autores como uma forma alternativa eficaz para o tratamento do estrabismo ${ }^{(1,5,8)}$.

A maioria dos trabalhos publicados relata a eficácia da 
toxina para determinado tipo de desvio ocular. Não foi encontrado na literatura qualquer trabalho que selecionasse os pacientes pelo tamanho do desvio ocular, apesar de existirem alguns que relatem melhores resultados com desvios de pequenos e médios ângulos ${ }^{(3,9-10)}$. Também não se encontrou qualquer estudo que comparasse a injeção unilateral com a bilateral, apesar de alguns autores relatarem os resultados com a injeção bilateral ${ }^{(1,9,11-12)}$. Resolvemos, então, comparar as três formas de tratamento, dividindo os pacientes em três grupos que foram considerados homogêneos pela análise estatística. Não subdividimos o grupo III em cirurgias unilaterais e bilaterais, porque apesar de ser ainda um assunto polêmico, vários trabalhos comprovaram que não há diferenças estatisticamente significativas entre as duas modalidades de cirurgia para a maioria dos desvios oculares ${ }^{(13)}$.

A comparação entre a evolução da medida do desvio horizontal, para longe e para perto, dos percentuais de correção do desvio e do índice de sucesso terapêutico entre os três grupos, mostrou que eles foram semelhantes, não havendo diferenças estatisticamente significativas.

Nos cálculos dos percentuais de correção e do índice de sucesso terapêutico, foram estudadas as medidas tomadas antes do tratamento e após três meses da realização deste, por se considerar que após o terceiro mês a medida do desvio ocular já não é mais influenciada apenas pelo tipo do tratamento a que o paciente foi exposto, mas também por fatores como presença ou ausência de boa amplitude fusional, falta de uso da correção óptica adequada, presença de ambliopia, disfunção de músculos oblíquos, presença de DVD descompensada, entre outros. Além disso, considerou-se que para os grupos I e II, após os três meses, já não havia qualquer efeito da toxina injetada ${ }^{(7,14)}$.

O grupo I apresentou percentual de correção do desvio ocular de $50,4 \%$, o grupo II de $55,8 \%$ e o grupo III de $77,0 \%$. As diferenças não foram estatisticamente significativas. Na literatura, observa-se percentual em torno de $60 \%$ para o tratamento com a toxina ${ }^{(14)}$. Para comparar esses dados, temos que observar que foi feita apenas uma injeção de toxina em cada paciente, com exceção de dois pacientes em que foram feitas duas injeções, mas cujos tratamentos foram considerados como isolados, pois a segunda injeção foi feita após três meses de observação da primeira, o que está de acordo a literatura ${ }^{(15)}$. Vários trabalhos consideram que para a obtenção do alinhamento ocular (desvio residual de até 10 DP), $40 \%$ dos pacientes necessitam de uma segunda aplicação e $20 \%$ de uma terceira ${ }^{(3,11,16)}$.

O índice de sucesso para correção do desvio para longe entre os três grupos foi equivalente, não havendo diferenças estatisticamente significativas. O do grupo I foi de $43,5 \%$, o do grupo II de $38,5 \%$ e o do grupo III de $60,9 \%$. Comparando-se esses índices com os dados da literatura, observa-se que apesar de os índices dos grupos I e II serem relativos a uma única injeção em cada paciente, são semelhantes aos de ou-

\begin{tabular}{|c|c|c|}
\hline \multicolumn{3}{|c|}{ Tabela 5} \\
\hline $\begin{array}{l}\text { Desvios } \\
\text { pré-injeção (DP) }\end{array}$ & $\begin{array}{l}\text { Doses } \\
\text { injetadas }\end{array}$ & $\begin{array}{c}\text { Desvios } \\
\text { pós-injeção ( } 3^{\circ} \text { mês) }\end{array}$ \\
\hline $\begin{array}{l}\text { XT } 20+H T \text { D/E } 6 \\
\text { XT' } 15+\text { HT' D/E } 6\end{array}$ & $5 U$ RLD & 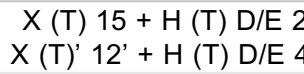 \\
\hline $\begin{array}{l}\text { ET } 25+H T \text { D/E } 15 \\
\text { ET' } 25\end{array}$ & $5 U$ RMS & $\begin{array}{l}E(T) 12+H(T) D / E \\
E(T)^{\prime} 12+H\left(T^{\prime}\right) D / E\end{array}$ \\
\hline $\begin{array}{l}\text { ET } 25 \\
\text { ET' } 25\end{array}$ & 5U RMS & $\begin{array}{l}E(T) 12 \\
E\left(T^{\prime}\right) 12\end{array}$ \\
\hline $\begin{array}{l}\text { ET } 25 \\
\text { ET' } 25\end{array}$ & 2.5U RMS & $\begin{array}{l}E(T) 12 \\
E\left(T^{\prime}\right) 15\end{array}$ \\
\hline $\begin{array}{l}\text { ET } 20 \\
\text { ET' } 20\end{array}$ & 5U RMD & $\begin{array}{l}E(T) 15 \\
E\left(T^{\prime}\right) 20\end{array}$ \\
\hline $\begin{array}{l}E(T) 15 \\
E(T), 20\end{array}$ & 5U RME & $\begin{array}{l}E(T) 15 \\
E\left(T^{\prime}\right) 18\end{array}$ \\
\hline $\begin{array}{l}\text { ET } 25 \\
\text { ET' } 12\end{array}$ & $5 U$ RMD & $\begin{array}{l}E(T) 15 \\
E\left(T^{\prime}\right) 12\end{array}$ \\
\hline $\begin{array}{l}\text { ET } 30+\text { HT D/E } 6 \\
\text { ET' } 25+\text { HT D/E } 6\end{array}$ & $5 U$ RME & 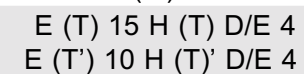 \\
\hline
\end{tabular}

tros autores: $\operatorname{Scott}^{(1)}$ relatou um índice de 57\%; Maggon $^{(17)}$, um índice de $35 \%$; e também, segundo Maggon ${ }^{(17)}$, Biglan, um índice de $38 \%$; e Elston, de $46 \%$.

É importante mencionar os casos nos quais a aplicação de toxina não diminuiu o desvio ocular para $10 \mathrm{DP}$ ou menos, mas significou melhora acentuada do ponto de vista estético, e, às vezes, também funcional. Foram casos em que desvios constantes se transformaram em tropias intermitentes, mesmo que o tamanho do desvio não estivesse dentro dos limites utilizados como critério para sucesso terapêutico. No $3^{\circ}$ mês após a aplicação da toxina, oito casos se transformaram em tropias intermitentes, sendo cinco pacientes do grupo I e três pacientes do grupo II, segundo a tabela 5.

\section{CONCLUSÃO}

Concluímos que a aplicação da toxina botulínica é uma forma alternativa válida para o tratamento dos estrabismos de pequeno e médio ângulos, e que não houve diferenças estatisticamente significativas entre os percentuais de correção do desvio ocular e nem do índice de sucesso terapêutico entre as três formas de tratamento.

\section{AB S T RAC T}

Purpose: To compare the efficacy of three types of treatment for strabismus: surgery, unilateral and bilateral botulinum toxin injections. Methods: To compose groups I and II, a prospective study was carried out observing the pretreatment measurement of the deviation, repeated after 7 , 30 and 90 days after the injection. To compose group III, a retrospective study was carried out. Results: The percentage of horizontal deviation correction for distance, in 3 months, 
was: group $\mathrm{I}=50.9 \%$; group $\mathrm{II}=55.8 \%$, and group $\mathrm{III}=$ $77.0 \%$; for near, $48.6 \%, 49.2 \%$ and $72.8 \%$ respectively. The therapeutic success rate, defined as residual deviation of 10 $\mathrm{PD}$ (prism diopter) or less, was: group $\mathrm{I}=57.1 \%$; group $\mathrm{II}=$ $68.4 \%$ and group $\mathrm{III}=72.4 \%$. Conclusions: There were no statisticaly significant differences between the percentage of deviation correction and success rate between the three types of treatment studied.

Keywords: Strabismus/surgery; Botulinum toxin type A; Case report

\section{REFERENCIAS}

1. Scott AB. Botulinum toxin injection of eye muscles to correct strabismus. Trans Am Ophthalmol Soc 1981;79:734-70.

2. Prieto-Díaz J, Souza-Dias C. Estrabismo. 2a ed. São Paulo: Roca; 1986. 440 p.

3. Almeida Santos E. Tratamento farmacológico. In: Souza-Dias CR, Almeida HC de. Estrabismo- Conselho Brasileiro de Oftalmologia. São Paulo: Roca; 1993. p. 105-11.

4. Santos EA. Toxina botulínica. Arq Bras Oftalmol 1997;60:547-8.

5. Tejedor J, Rodriguez JM. Retreatment of children after surgery for acquired esotropia: reoperation versus botulinum injection. Br J Ophthalmol 1998;82: 110-4.

6. Scott AB, Rosembaum A, Collins CC. Pharmacologic weakening of extraocular muscles. Invest Ophthalmol 1973;12:924-7.

7. Lennerstrand G, Nordb OA, Tian S, Eriksson-Derouet B, Ali T. Treatment of strabismus and nystagmus with botulinum toxin type A. An evaluation of effects and complications. Acta Ophthalmol Scand 1998;76:27-37.

8. Melling J, Hambleton P, Shone CC. Clostridium botulinum toxins: nature and preparation for clinical use. Eye 1988;2:16-23.

9. Gómez-de-Liaño R, Gómez-De-Liaño P, Rodriguez JM. Toxina botulínica. In: Barbosa, PH.. Estrabismo. Rio de Janeiro: Cultura Médica; 1997. p. 29095. [Biblioteca Brasileira de Oftalmologia].

10. Murray T. Strabismus: challenges and trends. Eye 1993;7(Pt3):332-40.

11. McNeer KW, Tucker MG, Spencer RF. Botulinum toxin management of essential infantile esotropia in children. [commented on Arch Ophthalmol 1997;115:1458-9]. Arch Ophthalmol 1997;115:1411-8.

12. Spencer RF, Tucker MG, Choi RY, Mcneer KW. Botulinum toxin management of childhood intermittent exotropia. Ophthalmology 1997;104:1762-7.

13. Alvares MG, Almeida HC. Correção das esotropias: cirurgia mono ou binocular. Rev Bras Oftalmol 1984;43:133-6.

14. Elston JS, Lee JP, Powell CM, Hogg C, Clark P. Treatment of strabismus in adults with botulinum toxin A. Br J Ophthalmol 1985;69:718-24.

15. Abbasoglu OE, Senner EC, Sanac AS. Factors influencing success and doseeffect relation of botulinum A treatment. Eye 1996;10(Pt3):385-91.

16. Ing, MR. Botulinum alignment for congenital esotropia.[commented on Ophthalmology 1993;100:1119]. Ophthalmology 1993;100:318-22.

17. Magoon EH. Botulinum chemodenervation for strabismus and other disorders. Int Ophthalmol Clin 1986;25:149-59.

\title{
VII CONGRESSO INTERNACIONAL DE CATARATA E CIRURGIA REFRATIVA
}

\section{6 a 9 de Abril de 2002}

\section{Hotel Transamérica - São Paulo - SP}

\author{
Organização: Sociedade Brasileira de Catarata e Implantes Intraoculares \\ e \\ Sociedade Brasileira de Cirurgia Refrativa
}

Informações: JDE Comunicação e Eventos

Alameda Santos, 1.343 - Ci. 304

Cep 01419-001 - São Paulo - SP

Telf.: (11) 287-8109 / 287-9699 - Fax: 288-8157

E-mail: jdecomev@uol.com.br 